Mathématiques et sciences humaines
Mathematics and social sciences

159 | Automne 2002

Varia

\title{
Les mathématiques de la population, de Lambert à
} Lotka

The mathematics of population from Lambert to Lotka

Jacques Veron

\section{(2) OpenEdition}

1 Journals

Édition électronique

URL : http://journals.openedition.org/msh/2868

DOI : $10.4000 / \mathrm{msh} .2868$

ISSN : 1950-6821

Éditeur

Centre d'analyse et de mathématique sociales de l'EHESS

Édition imprimée

Date de publication : 1 septembre 2002

ISSN : 0987-6936

Référence électronique

Jacques Veron, "Les mathématiques de la population, de Lambert à Lotka », Mathématiques et sciences humaines [En ligne], 159 | Automne 2002, mis en ligne le 10 février 2006, consulté le 23 juillet 2020. URL : http://journals.openedition.org/msh/2868; DOI : https://doi.org/10.4000/msh.2868

〔c École des hautes études en sciences sociales 


\title{
LES MATHÉMATIQUES DE LA POPULATION, DE LAMBERT À LOTKA
}

\author{
Jacques VÉRON ${ }^{1}$
}

RÉSUMÉ - En 1825, Benjamin Gompertz propose une formulation mathématique de la loi de mortalité, qui, comme celle de Lambert (1772), lie la survie à l'âge. En 1844, Pierre-François Verhulst propose un modèle de croissance d'une population tel que le taux d'accroissement diminue quand l'effectif progresse : c'est la fonction logistique (Lotka, à partir de 1907, contribuera largement à ce champ de la dynamique des populations et s'intéressera notamment à la stabilité de la structure par âge d'une population). Au XIX siècle encore, Wilhelm Lexis estime la durée normale de la vie humaine, durée que l'on observerait en l'absence de décès prématurés, dans l'enfance et au cours de la vie adulte.

MOTS-CLÉS - Loi de mortalité, Vie moyenne, Vie probable, Vie normale, Loi logistique, Population malthusienne, Population à répartition stable par âge.

SUMMARY - The Mathematics of Population from Lambert to Lotka

In 1825, Benjamin Gompertz gives a mathematical formulation of the law of mortality, which, following a former one by Lambert (1772), relates survival to age. In 1844, Pierre-François Verhulst put forward a model of population growth in which the rate of growth reduces when the size of the population increases: it is the logistic function (Lotka will, from 1907, contribute largely to this field of population dynamics, especially on the stability of the age composition of a population). During the $19^{\text {th }}$ century too, Wilhelm Lexis gives estimations of the normal length of human life, which would be observed in the absence of premature deaths, in childhood and adulthood.

KEYWORDS - Law of mortality, Mean length of life, Median length of life, Normal Length of Life, Logistic law, Malthusian Population, Population with a Stable Age Distribution.

La mathématisation de la science de la population progresse au XIX ${ }^{\mathrm{e}}$ siècle, notamment grâce la formulation par Benjamin Gompertz d'une loi de la mortalité fondée sur l'âge et par Pierre-François Verhulst de la loi logistique, selon laquelle la croissance de la population se ralentit du fait de cette croissance même.

L'analyse du processus de la mortalité et la dynamique des populations ont en réalité fait l'objet d'une attention particulière dès le XVII ${ }^{\mathrm{e}}$ siècle, de la part de ceux qui se sont intéressés aux phénomènes de population : différents auteurs se sont demandé quel parti tirer de l'approche probabiliste pour rendre compte de la survie humaine

\footnotetext{
${ }^{1}$ Institut national d'études démographiques, 133 boulevard Davout 75980 Paris cedex 20, e-mail : veron@ined.fr.
} 
tandis que d'autres ont, par exemple, fait porter leur attention sur le doublement d'une population.

Cette présentation des mathématiques de la population se limite au XIX ${ }^{\mathrm{e}}$ siècle mais il nous a paru utile de «déborder » quelque peu sur les siècles précédents et suivants. Les travaux du mathématicien Lambert, dans la seconde moitié du XVIII siècle, sont en effet intéressants à rapprocher de ceux de Gompertz. La dynamique des populations progresse grâce à Verhulst au milieu du XIX ${ }^{\mathrm{e}}$ siècle mais elle connaît un développement remarquable, dès le tout début du $\mathrm{XX}^{\mathrm{e}}$ siècle, sous l'impulsion notamment d'Alfred J. Lotka.

\section{ESSAIS DE MATHÉMATISATION DE LA LOI DE MORTALITÉ}

Le traitement mathématique ou statistique de la mortalité n'est possible que s'il existe un ordre de la mortalité, c'est-à-dire si la mort ne relève pas seulement de destins singuliers mais obéit à des lois. Comment peut alors intervenir le hasard ? La mort ne se distribue pas au hasard dans le sens où il n'y a pas une équiprobabilité de décéder à chaque âge. Si la mortalité croît exclusivement avec l'âge, le hasard semble pouvoir être en partie éliminé. Mais peut-être faut-il combiner les effets de l'âge et ceux du hasard pour rendre compte de la mortalité. Le hasard peut aussi se présenter sous la forme d'aléas autour d'une valeur centrale, la durée normale de la vie.

\subsection{L'ÉQUATION DE LAMBERT (1772)}

Mathématicien, il donne la première démonstration de l'irrationalité de $\square$, et philosophe, il publie en 1764 Neues Organon et correspond notamment avec Kant. Johann-Heinrich Lambert présente dans ses Contributions à l'utilisation des mathématiques et à leur application $^{2}$ - trois volumes dont la publication s'échelonne de 1765 à 1772 d'importants travaux, notamment sur la mortalité.

Dans le premier volume de ces contributions, Lambert se demande comment tracer une courbe à main levée qui ajuste au mieux les données de mortalité, dans le cas d'espèce, celles tirées des registres de mortalité de Londres (1753-1758). Dans le troisième volume, un mémoire entier est consacré aux questions démographiques (mémoire $\mathrm{n}^{\circ}$ 9), il s'agit des « Remarques sur la mortalité, les listes de décès, de naissances et de mariages $»^{3}$.

Lambert propose dans ce mémoire une équation de la mortalité, rendant compte de l'évolution de la survie d'une population en fonction de son âge (Figure 1) $: \frac{7}{10} y$ représentant le nombre de survivants et $x$ l'âge.

\footnotetext{
${ }^{2}$ Beyträge zum Gebrauche der Mathematik und deren Anwendung.

${ }^{3}$ Anmerkungen über die Sterblichkeit, Todtenlisten, Geburthen und Ehen.

${ }^{4}$ Il est à noter une inversion de deux chiffres dans la première exponentielle, à la place de 31,682 on doit
} 


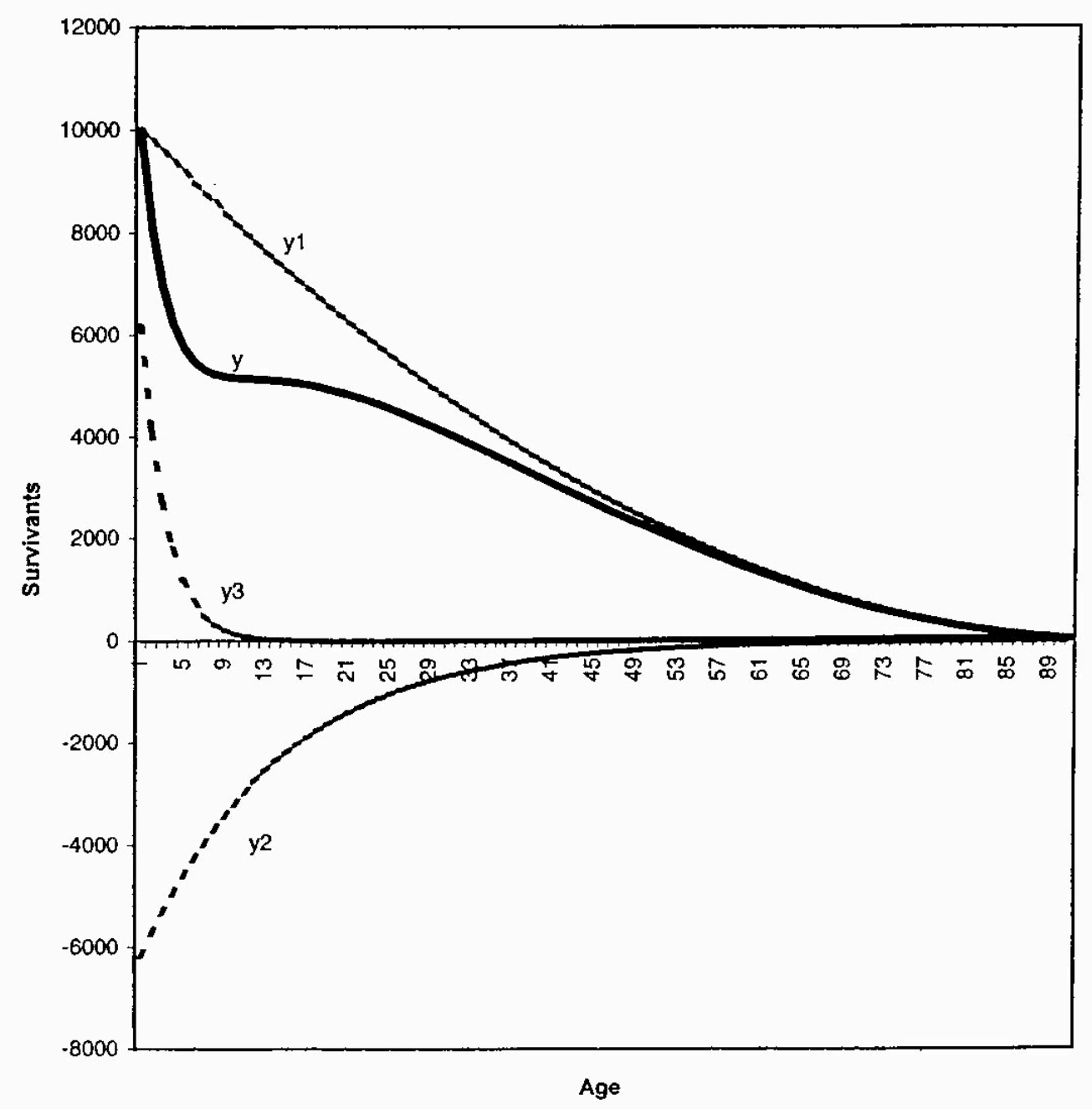

Figure 1. Courbe de mortalité tracée à partir de l'équation de Lambert

$$
(y=y 1+y 2+y 3)
$$

Cette équation permet un très bon ajustement des données de mortalité. Lambert justifie cette formulation de la loi de mortalité de manière surprenante : il s'agit d'une parabole (eine Parabel) et de deux lignes logistiques (zwei logistische Linien). La parabole, écrit-il, traduit le fait que l'espèce humaine s'éteint à la manière d'un récipient cylindrique qui se vide de son eau, tandis que les deux autres membres ont quelque chose de très analogue au réchauffement et refroidissement des corps.

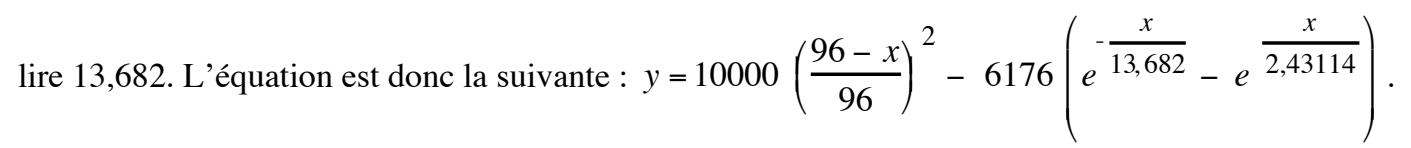


L'équation de Lambert constitue la première formulation d'une loi de survie en termes algébriques (Guiquet, 1893). Mais la formulation de la mortalité qui va devenir la référence, c'est celle proposée par Gompertz.

\subsection{LA LOI DE GOMPERTZ (1825)}

Benjamin Gompertz publie dans les Philosophical Transactions of the Royal Society of London, en 1825, un très long article intitulé "On the Nature of the Function Expressive of the Law of Human Mortality, and on a New Mode of Determining the Value of Life Contingencies », dans lequel il présente un modèle d'évolution de la mortalité selon l'âge.

Considérons la présentation que fait Albert Quiquet de la loi de Gompertz :

Étendant, en 1825, son premier mémoire [celui de 1820], Gompertz attribue la mort à deux grandes causes. La première, due au hasard seul, frappe sans préparer ses coups et avec autant d'intensité pendant la vieillesse que pendant la jeunesse : si elle était seule, les tables de survie et de mortalité seraient de simples progressions géométriques.

La seconde cause, au contraire, agit d'une façon continue pour affaiblir "notre résistance à la destruction" ; en d'autres termes elle accrô̂t "notre aptitude à mourir". Les survivants doivent donc diminuer plus vite que ne l'indique le taux d'une progression géométrique.

[...] Si à la fin d'intervalles de temps infiniment petits, "la résistance à la destruction" perd une fraction, proportionnelle à l'accroissement du temps, de la valeur qu'elle avait au commencement de ces intervalles, alors le "pouvoir contre la mort", ou "l'intensité de la mortalité" qui lui est inverse se mettra sous la forme $\mathrm{aq}^{\mathrm{x}}$; le nombre - $\mathrm{dl}(\mathrm{x})$ des décès dans le temps $\mathrm{dx}$ sera égal à

d'où

$$
\square d l(x)=l(x) \cdot a q^{x} d x
$$

$$
l(x)=k^{q^{x}}
$$

Telle est la célèbre loi de Gompertz, avec les considérations qui l'y ont amené $^{5}$.

Comme Lambert, Gompertz établit un parallèle entre mortalité et physique, l'intensité de la mortalité s'exprimant comme « la diminution de la force élastique dans une machine pneumatique au bout de $x$ coups de piston. » (cité par Quiquet, p. 15).

En marge des travaux des actuaires, une des approches de la mortalité continue de privilégier la distinction entre vie moyenne et vie probable.

\subsection{MARCHE COMPARATIVE DES VIES MOYENNE ET PROBABLE}

L'annuaire de la Statistique de la France de 1857 donne l'exemple d'une approche très descriptive de la loi de mortalité : il s'agit essentiellement de rendre compte de la

\footnotetext{
${ }^{5}$ A. Quiquet, 1893, p. 14-15.
} 
« survivance » d'une population en consignant dans un tableau la mortalité relative à chaque âge.

On trouve par ailleurs une présentation de la «marche comparative des vies moyenne et probable » (Tableau 1) qui permet de constater que, à la naissance, vie moyenne et vie probable s'égalisent pratiquement ( 2 mois d'écart seulement), alors que, dès l'âge d'un an, une grande différence apparaît (près de 5 années). Cette différence décroît ensuite pour s'annuler pratiquement à l'âge de 50 ans.

Tableau 1. Marche comparative des vies moyenne et probable en 1853 et 1854

\begin{tabular}{|c|c|c|c|c|c|c|c|c|c|c|c|c|c|}
\hline \multirow{3}{*}{$\begin{array}{c}\text { Ages } \\
\text { Naissance } \\
1 \text { an }\end{array}$} & \multicolumn{3}{|c|}{ Vie moyenne } & \multicolumn{3}{|c|}{ Vie probable } & \multicolumn{3}{|c|}{ Différence } & \multicolumn{4}{|c|}{$\begin{array}{c}\text { Différence de la vie } \\
\text { moyenne et de la vie } \\
\text { probable en } 1853\end{array}$} \\
\hline & \multicolumn{3}{|c|}{37 ans 0 mois } & \multicolumn{3}{|c|}{37 ans 2 mois } & \multicolumn{3}{|c|}{+0 ans 2 mois } & \multicolumn{2}{|c|}{+1 an } & \multicolumn{2}{|c|}{0 mois } \\
\hline & & -4 & - & & -0 & - & +4 & -8 & & +6 & - & 3 & \\
\hline 3 ans & 46 & -5 & - & 51 & -3 & - & +4 & -10 & - & +6 & - & 1 & - \\
\hline 10 ans & 43 & -9 & - & 47 & -6 & - & +3 & -9 & - & +4 & - & 9 & - \\
\hline 20 ans & 36 & -11 & - & 39 & -1 & - & +3 & -0 & - & +3 & - & 10 & - \\
\hline 30 ans & 31 & -7 & - & 33 & -6 & - & +1 & -11 & - & +2 & - & 6 & - \\
\hline 40 ans & 25 & -2 & - & 26 & -3 & - & +1 & -1 & - & +1 & - & 5 & - \\
\hline 50 ans & 18 & -8 & - & 18 & -11 & - & +0 & -3 & - & +0 & - & 6 & - \\
\hline 60 ans & 12 & -11 & - & 12 & -5 & - & +0 & -6 & - & +0 & - & 5 & - \\
\hline 70 ans & 7 & -10 & - & 6 & -9 & - & -1 & -1 & - & -1 & - & 0 & - \\
\hline 80 ans & 4 & -7 & - & 3 & -9 & - & -0 & -10 & - & -0 & - & 10 & - \\
\hline 90 ans & 2 & -11 & - & 2 & -3 & - & -0 & -8 & - & -0 & - & 7 & - \\
\hline
\end{tabular}

\subsection{AGES ET VIE MOYENNE}

La mortalité peut être envisagée, non sous l'angle de relations fonctionnelles, entre survie et âge par exemple, mais de manière purement numérique. C'est l'approche adoptée par G. de Serbonnes dans Le Moniteur des Assurances (1875).

Il écrit ainsi :

[...] la vie moyenne entre 20 et 60 ans, période active de l'existence, semble régie par une loi dont la clé est le nombre cabalistique 7.

Serbonnes met en évidence, dans les données auxquelles il se réfère ${ }^{6}$ (Tableau 2), une progression arithmétique décroissante de raison $\frac{7}{10}$ :

\footnotetext{
${ }^{6}$ Il s'agit des données de « la table anglaise dite de la nouvelle expérience ».
} 
On a ainsi, en partant de 42, qui représente la vie moyenne à 20 ans, la progression suivante dont la raison est $\frac{7}{10}$ :

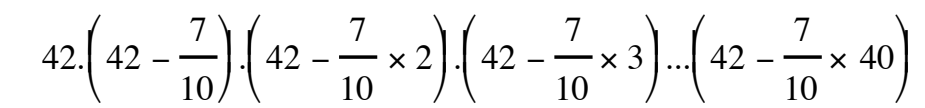

Il s'ensuivrait donc que nous userions chaque année $\frac{7}{10}$ d'année, ou 255 jours et $\frac{1}{2}$ de notre vie moyenne.

Tableau 2. Evolution de la vie moyenne selon l'âge

\begin{tabular}{|c|c|}
\hline $\begin{array}{c}\hat{\text { Age }} \text { (en années) } \\
\text { (en }\end{array}$ & $\begin{array}{c}\text { Vie moyenne } \\
\text { (en années) }\end{array}$ \\
\hline 20 & 42 \\
30 & 35 \\
40 & 28 \\
50 & 21 \\
60 & 14 \\
\hline
\end{tabular}

Serbonnes donne une formule permettant de calculer l'espérance de vie à n'importe quel âge :

D’une façon générale, si nous appelons $A$, un âge quelconque, la vie moyenne à cet âge sera représentée par :

$$
42 \square \frac{7 \square(A \square 20)}{10}
$$

ou

$$
\underline{420 \square 7 \square(A \square 20)}
$$

10

et en observant que 420 est un multiple de 7

$$
\frac{7 \square(60+20 \square A)}{10}
$$

ce qui donne la formule plus simple :

$$
\frac{7 \square(80 \square A)}{10} \text {. }
$$

Très différente de l'approche de la mortalité par Serbonnes est celle du statisticien allemand Wilhelm Lexis, pour lequel il existe un ordre naturel de la mortalité. 


\subsection{LE CONCEPT DE VIE NORMALE : LEXIS $(1877,1878)$}

Lexis présente au premier Congrès international de Démographie qui se tient à Paris en 1878 , une communication portant «Sur la durée normale de la vie humaine et sur la théorie de la stabilité des rapports statistiques ».

Pour le statisticien allemand, comme pour Quetelet, il existe un modèle unique de l'homme et les êtres humains en sont des « copies plus ou moins exactes ».

En considérant un grand nombre de personnes, remarque Lexis, on peut se faire une idée de ce modèle unique de l'homme, par concentration des cas autour d'une valeur particulière :

[...] les écarts individuels de ce type, pris en grands nombres, se groupent autour de la moyenne, d'après la loi bien connue des erreurs accidentelles.

À ce modèle unique de l'homme correspond le «type normal de la vie humaine » :

[...] dans toute génération supposée assez nombreuse, un certain groupe réalisera dans sa vie moyenne le type normal avec les écarts conformes à la formule appelée par Qu[é]telet la loi du binôme.

Lexis regroupe l'ensemble des durées de vie individuelles en trois sousensembles (cf. Figure 2) :

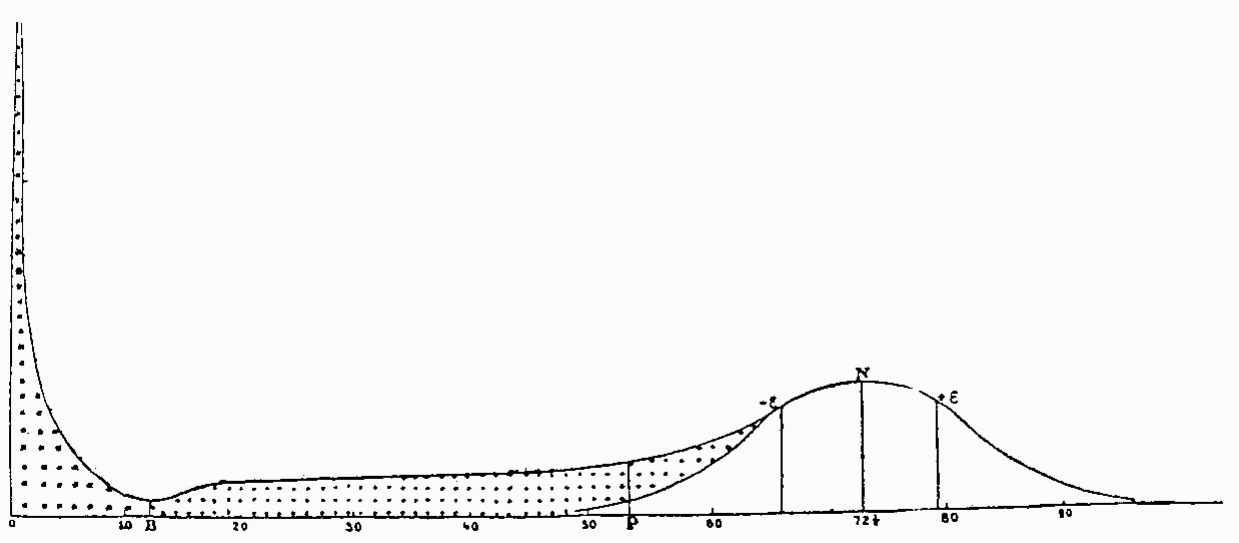

Figure 2. Courbe des décès de Lexis

- l'ensemble des durées de vie du «type normal »;

- l'ensemble des durées de vie des enfants morts en bas âge ;

- l'ensemble des durées de vie des «autres individus ayant succombé à une mort prématurée et à peu près indépendante du degré d'âge ». 
Dans le groupe normal, la dispersion des âges au décès obéit exclusivement à la loi des erreurs accidentelles. En revanche, les âges au décès du groupe des enfants morts en bas âge n'ont, selon Lexis, rien à voir avec la durée normale de la vie humaine :

[ce groupe] n'est pas même entré en concurrence pour atteindre les limites naturelles de la vie.

Pour estimer la mortalité normale, il faut pouvoir établir une frontière entre groupes adulte et normal des décès. Lexis se sert des données françaises de la mortalité, rassemblées par Jacques Bertillon.

Les décès masculins sont les plus nombreux dans le groupe d'âge 70-75 ans (Tableau 3). Lexis considère que la valeur centrale de ce groupe d'âge, soit 72 ans 1/2, est l'âge normal au décès. Il suppose par hypothèse que tous les décès qui se produisent au-delà de l'âge normal sont des « décès normaux », ce qui lui permet par symétrie d'estimer le nombre de décès normaux avant l'âge de 72 ans 1/2.

Tableau 3. Extrait de la table de mortalité de Jacques Bertillon pour les hommes ${ }^{7}$, reproduite par Wilhelm Lexis

\begin{tabular}{|c|c|c|}
\hline \multicolumn{3}{|c|}{ Hommes } \\
\hline Morts à l'âge de & d'après la table & d'après la théorie \\
\hline 45 à 50 ans & 16 & $(2)$ \\
$50-55$ & 19 & $(4)$ \\
$55-60$ & 24 & $(12)$ \\
$60-65$ & 32 & $(24)$ \\
\hline $65-70$ & 38 & 37 \\
$70-721 / 2$ & $\underline{20}$ & $\underline{21}$ \\
\hline $721 / 2-75$ & 20 & 21 \\
$75-80$ & 38 & 24 \\
$80-85$ & 26 & 12 \\
$85-90$ & 12 & 6 \\
\hline Au dessus de 90 & 4 & \\
\hline \multicolumn{2}{|c|}{ Source : Lexis, 1878, p. 151 } \\
\hline
\end{tabular}

Lexis résume finalement la mortalité des hommes en France à l'aide de trois nombres :

- un âge normal au décès de 72 ans $1 / 2$;

- une proportion de $40 \%$ d'hommes appartenant au groupe normal des décès ;

- un écart probable, centré sur l'âge normal, de 6,275 ans (c'est-à-dire $50 \%$ des hommes décèdent entre les âges de 72,5 - 6,275 ans et 72,5 + 6,275 ans).

Lexis considère que la « concentration autour du centre de densité (à 72 ans 1/2) 》 représente « le but que la nature tend à atteindre et dont elle s'écarte dans l'un et l'autre sens d'après la loi des erreurs accidentelles. »

\footnotetext{
${ }^{7}$ Année non précisée.
} 
La dynamique des populations constitue un deuxième axe de mathématisation de la science de la population.

\section{2. ÉLEMENTS DE DYNAMIQUE DES POPULATIONS}

\subsection{La CROISSANCE LOGistiQue : Verhulst (1844)}

Selon Malthus (1798), en l'absence de limitation par la quantité de ressources disponibles, toute population croîtrait selon une progression géométrique. Se fondant sur l'expérience américaine, où la population n'était pas freinée à la fin du XVIII ${ }^{\mathrm{e}}$ car il y avait alors abondance de nourriture, Malthus estimait que le rythme de croissance pouvait être tel que, si elle n'était pas freinée, la population doublait en 25 années.

En référence à la conception malthusienne de la puissance de peuplement, on dénomme aujourd'hui « malthusienne ${ }^{8}$ une population qui croît à un taux constant :

$$
\frac{d P}{P . d t}=r
$$

Dans ce cas la croissance est exponentielle, puisqu'en intégrant on obtient l'égalité suivante :

$$
P(t)=P(0) e^{r t}
$$

Pierre-François Verhulst, qui s'était intéressé à la théorie de la population sous l'impulsion de Quetelet [Schtickzelle, 1981] propose un autre modèle de croissance d'une population que celui proposé par Malthus, celui où la population verrait sa croissance se ralentir au fur et à mesure que son effectif progresse. Verhulst donne en 1844 le nom de logistique à cette fonction.

Le taux de croissance de la population est de la forme suivante ${ }^{9}$ :

$$
\frac{d P(t)}{P . d t}=r \text { 目 } 1 \frac{P(t)}{a} \text { 目 }
$$

$a$ est tel que si $P(t)=a$, la croissance est nulle.

L'égalité (1) peut aussi s'écrire :

$$
\frac{1}{P(t)}+\frac{1}{a \square P(t)}-\mathrm{B} d P(t)=r d t
$$

\footnotetext{
${ }^{8}$ Une « population malthusienne » est soit une population qui croît à taux constant (par conséquent une population dont la croissance est exponentielle), soit une population dans laquelle les couples pratiquent une limitation des naissances (c'est-à-dire au sein de laquelle s'est répandue la contraception).

${ }^{9}$ Nous empruntons la présentation à Nathan Keyfitz (1985).
} 
qui donne après intégration :

$$
\text { In } \left.\frac{P(t)}{a \square P(t)}\right]=r t+c
$$

et en posant, $t_{0}=\square \frac{c}{r}$, afin de situer le point moyen de la courbe en $\mathrm{t}_{0}$, on obtient :

$$
P(t)=\frac{a}{1+e^{\square r\left(t \square t_{0}\right)}}
$$

Pearl et Reed (1920) ont proposé, sans connaître les travaux de Verhulst, la formulation d'une courbe logistique, mais sans la nommer ainsi. Ils la décrivaient ainsi :

[...] une courbe de population peut commencer par une convexité par rapport à la base, mais ensuite elle doit avoir un point d'inflexion, et à partir de ce point présenter une concavité par rapport à l'axe des $x$, puis finalement devenir asymptotique, l'asymptote représentant le nombre maximal de personnes qu'un territoire donné de dimension fixée peut nourrir... ${ }^{10}$

Notons que Pearl et Reed avaient ainsi déterminé un effectif maximal pour la population des États-Unis de 197270000 personnes (la population actuelle est supérieure à 280 millions).

Si la fonction logistique est intéressante du point de vue de la dynamique des populations, cette partie de la science de la population ne progresse véritablement qu'au début du XX $X^{\mathrm{e}}$ siècle, notamment sous l'influence de Lotka.

\subsection{Populations MalthusienNeS ET STABLES : LotKA}

Alfred James Lotka présente, dans un article de 1907, deux équations devenues fondamentales de la dynamique des populations.

Ainsi, après avoir noté $b$ le taux de natalité par tête, $d$ le taux de mortalité par tête, $c(a)$ la proportion de personnes d'âge compris entre les âges $a$ et $(a+d a), p(a)$ la probabilité à la naissance d'atteindre l'âge $a, B_{(t-a)}$ le nombre de naissances au temps $t$ - $a$ et $N_{t}$ le nombre de personnes en $t$, Lotka pose l'égalité suivante, qui concerne le nombre de survivants à l'âge $a$ :

$$
N_{t} c(a) d a=B_{(t \square a)} P(a) d a
$$

ou encore :

$$
c(a)=\frac{B_{t \sqcap a}}{N_{t}} p(a)
$$

\footnotetext{
${ }^{10}$ Cf. Smith et Keyfitz, 1977, p. 342 (la traduction est de l'auteur de cet article).
} 
Si les conditions générales sont constantes (la structure par âge est notamment fixe), le taux de croissance $r$ est lui-même constant $(r=b-d)$. Les naissances augmentent alors en progression géométrique et on peut écrire :

$$
\begin{gathered}
B_{(t \square a)}=B_{t} e^{\square r a} \\
c(a)=\frac{B_{t}}{N_{t}} e^{\square r a} p(a)
\end{gathered}
$$

et finalement :

$$
c(a)=b e^{\square r a} p(a)
$$

Comme on a, par définition de $c(a)$ :

$$
\square_{0} c(a) d a=1
$$

On peut écrire :

$$
\frac{1}{b}=\underset{0}{\square} e^{\square r a} p(a) d a
$$

L'équation (1) donne la structure par âge. L'équation (2) précise la relation entre le taux de natalité et le taux de croissance, mais aussi, comme le taux de croissance est égal à la différence entre taux de natalité $b$ et taux de mortalité $d$, la relation entre taux de natalité et taux de mortalité.

Dans un article de 1911 écrit avec F. R. Sharpe ainsi que dans un autre datant de 1922, Lotka examine le caractère «stable» de la distribution par âge d'une population $^{11}$ : il s'agit de savoir si, après avoir subi des perturbations, la structure par âge tend à revenir à celle, fixe, que caractérise l'équation (1).

Lotka parle de «population malthusienne » lorsque la mortalité et la composition par âge sont invariables (le taux de croissance étant constant, la croissance de la population suit « la loi de Malthus »). Il parle de «population à répartition stable par âge $»^{12}$ lorsque, outre ces deux premières conditions, la fécondité est supposée constante.

Si $m(a)$ représente la «fécondité effective » à l'âge $a^{13}$, une autre égalité se vérifie alors :

\footnotetext{
${ }^{11}$ Dans sa Théorie analytique des associations biologiques (1934, 1939), Lotka présente une synthèse de ces questions.

${ }^{12}$ Ce thème de la stabilité d'une population avait déjà été abordé, notamment par Euler dans ses «Recherches générales sur la mortalité et la multiplication du genre humain » (1760). Cf. BourgeoisPichat, 1994.

${ }^{13}$ Pour plus de commodité, Lotka raisonne sur la population de sexe féminin. Le nombre $B(t)$ est, dans ce cas, celui des naissances annuelles de filles. La fécondité effective $m(a)$ est, elle, définie comme «le nombre de filles nées vivantes par an per capita de femmes d'âge $a$. » [Lotka, 1939, p. 64].
} 


$$
\square_{0}^{\square e^{\square r a}} p(a) m(a) d a=1
$$

À quelques travaux près, comme ceux de Gompertz ou de Verhulst par exemple, la démographie reste, à la fin du XIX ${ }^{\mathrm{e}}$ siècle, encore très descriptive. Ainsi, dans un ouvrage de référence tel que La population française de Levasseur (le tome premier date de 1889 et le tome deuxième de 1891), ouvrage dans lequel il est notamment affirmé que «[l]a statistique est l'étude numérique des faits sociaux», l'accent est mis sur les moyennes et les rapports et sur la régularité des phénomènes démographiques. Mais, dans cet ouvrage, Levasseur se livre avant tout à une description de la natalité, de la nuptialité et de la mortalité sans rechercher des tendances particulière susceptibles d'être assimilées à des lois.

La mathématisation de la science de la population date véritablement du $\mathrm{XX}^{\mathrm{e}}$ siècle. Lotka a donné une forte impulsion à la dynamique des populations ; parmi ses continuateurs, on peut notamment mentionner Ansley J. Coale (1972), Jean BourgeoisPichat (1994) et Nathan Keyfitz (1985).

\section{RÉFÉRENCES}

[* L'astérisque accompagnant un nom d'auteur signifie que le texte en question a été repris, partiellement ou complètement, dans l'ouvrage de David Smith et Nathan Keyfitz, Mathematical Demography, Springer-Verlag, Berlin, 1977].

BOURGEOIS-PICHAT J., La dynamique des populations : populations stables, semistables et quasi-stables, Paris, Presse Universitaire de France, INED, 1994.

COALE A. J., «The Growth and Structure of Human Populations », A Mathematical Investigation, Princeton, University Press, 1972.

GOMPERTZ* B., «On the Nature of the Function Expressive of the Law of Human Mortality, and on a New Mode of Determining the Value of Life Contingencies », Philosophical Transactions of the Royal Society of London, Volume 115, 1825, p. 513583.

KEYFITZ N., Applied mathematical Demography, second edition, New York, SpringerVerlag, 1985.

LAMBERT J. H., Beiträge zum Gebrauche der Mathematik und deren Anwendung, Berlin, Dritter Theil, 1772.

LOTKA* A. J., «Relation Between Birth Rates and Death Rates », Science N. S. 26, 1907, p. 21-22.

LOTKA* A. J. , «The Stability of the Normal Age Distribution », Science N. S. 26, 1922, p. 21-22. 
LOTKA A. J., Théorie analytique des associations biologiques, I : Principes, 1934 et II : Analyse démographique avec application particulière à l'espèce humaine, 1939, Paris, Hermann.

MALTHUS T. R., Essai sur le principe de population, Paris, INED, 1798.

PEARL* R. et REED L. J., «On the Rate of Growth of the Population of the United States since 1790 and its Mathematical Representation », Proceedings of the National Academy of Science 6, 1920.

QUICQUET A., Aperçu historique sur les formules d'interpolation des tables de survie et de mortalité, Deuxième édition, Paris, Warnier et $C^{\text {ie }}, 1893$.

SCHTICKZELLE M., « Pierre-François Verhulst (1804-1849). La première découverte de la fonction logistique », Population 3, 1981, p. 541-556.

SERBONNES G. (de), «La vie moyenne de la table $\mathrm{H}^{\mathrm{MF}}$ », Le moniteur des assurances, 1875.

SHARPE* F.R. and LOTKA A. J., «A Problem in Age-Distribution », Philosophical Magazine, Series 6, vol. 21, 1911, p. 435-438. 